\title{
TRACING MICROTUBULES IN LIVE CELL IMAGES
}

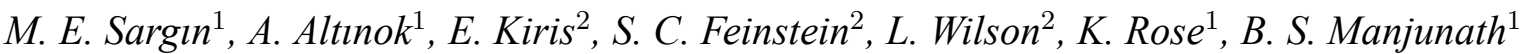 \\ Dept. of Electrical and Computer Engineering ${ }^{1}$, \\ Dept. of Molecular, Cellular, and Developmental Biology², \\ University of California Santa Barbara, Santa Barbara, CA 93106 \\ \{msargin,alphan\}@ece.ucsb.edu
}

\begin{abstract}
Microtubule (MT) dynamics are traditionally analyzed from time lapse images by manual techniques that are laborious, approximate and often limited. Recently, computer vision techniques have been applied to the problem of automated tracking of MTs in live cell images. Aside of very low signal to noise ratios, live cell images of MTs exhibit severe clutter for accurate tracing of MT body. Moreover, intersecting and overlapping MT regions appear brighter due to additive fluorescence. In this paper, we present a MT body tracing algorithm that addresses the clutter without imposing directional constraints. We show that MT dynamics can be quantified with enhanced precision, and novel measurements that are beyond manual feasibility, can be obtained accurately. We demonstrate our results on actual images of MTs obtained by live cell fluorescence microscopy.
\end{abstract}

Index Terms - Biomedical image processing, Image line pattern analysis, Object detection

\section{INTRODUCTION}

Microtubules (MTs) are filamentous cytoskeletal structures composed of tubulin protein subunits. These subunits can add on, or dissociate from, the tubulin polymer rapidly, making MTs highly dynamic. Through these dynamic behaviors, MTs are critically involved in many essential cellular functions, such as chromosome segregation at mitosis and intracellular cargo transport. The growing and shortening dynamics of MTs are finely regulated by well known regulatory mechanisms, for example, by the action of MT-associated proteins. For additional background on MT structure and function, see [1].

A large body of evidence, reviewed by Feinstein and Wilson [2] suggest that cell viability requires that MT dynamics be properly regulated within a narrow range of activities. Indeed, it has been hypothesized that inadequate regulation of neuronal MT dynamics may underlie neuronal cell death

This study was funded by Center for Bioimage Informatics under grants NSF-ITR 0331697, NIH-ROI NS35010, NIH-ROI NS13560. in Alzheimer's and related dementias. Additionally, drug induced modulation of MT dynamics underlies the effectiveness of various anti-cancer drugs, such as Taxol. For these and a host of basic biology issues, the regulation of MT dynamics is a very active area of research in modern cellular biology.

A key tool of MT dynamics research is to track individual MT tips from time lapse images, Fig.1, and quantitatively describe MT behavior under different experimental conditions. Traditional MT dynamics parameters consist of statistics derived from the growth and shortening events between consecutive frames. In general, tracking is a largely manual and laborious task. While MT body tracking is highly desired to assess MT behavior, it is beyond manual feasibility. For example, with accurate body tracking, it would be possible to quantitatively study MTs that take part in neuronal growthcone path finding. Recently suggested methods for automating MT tracking are limited to MTs in isolated areas. Tracking performance, especially body tracking, considerably degrades with intersecting and occasionally overlapping MTs, which are very common in live cell images of MTs. Therefore, automated tracking methods can immediately benefit from accurate tracing of individual MTs in live cell images.

In this paper we address the shortcomings due to clutter. In doing so, we avoid imposing curvature constraints on the MT body. Curvature based techniques, e.g. [3], are effective in tracing relatively isolated curvilinear structures. However, the main challenge of live cell MT images is the frequent intersections that cause intensity variations along the MT body because of additive fluorescence. Moreover, measuring curvature is one of the goals, which ideally should not be constrained. We demonstrate our tracing algorithm on actual MT image sequences obtained from [4] and [5], resulting in accurate traces.

The rest of this paper is organized as follows. In Section 2, we review past work and discuss associated problems. In Section 3, we describe the proposed tracing algorithm. In Section 4, we describe the dataset used in this work. In Section 5, we present our tracing results and conclude in Section 6 with discussion and future directions. 


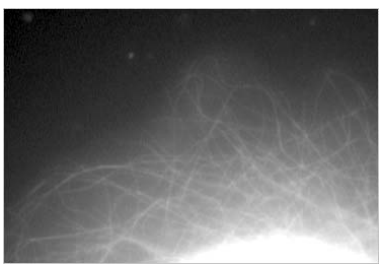

(a)

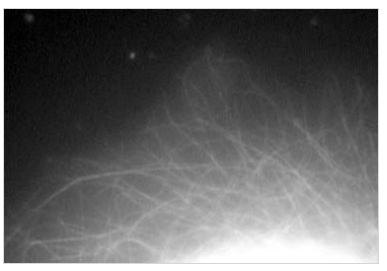

(b)
Fig. 1. Consecutive MT frames from time lapse images.

\section{RELATED WORK}

Depending on the exact experimental procedures and equipment, acquired MT images change considerably in quality and content. In this study, we consider only live cell image sequences acquired by fluorescence microscopy. This microscopy technique is preferred over newer alternatives, such as the laser scanning confocal microscopy, typically for faster acquisition speed. However, resulting images suffer from fluorescence aberrations and distortions in the optical path, Fig.2. Each frame represents a projection image, in which various MTs appear as intersecting. Furthermore, intersections may exhibit higher intensity levels. Thus, intensity variations on MT bodies are expected. The general problem of MT segmentation remains a very difficult task.

MTs appear as open ended curves in images, where one end (plus end) is visible and dynamic and the other end (minus end) is assumed to be fixed near the nucleus and not visible due to saturation, Fig.1. The MT shape is likely to be a result of interfering (but invisible) cellular organelles. Therefore, constrained curve models should not be preferred in tracing MT bodies.

Recent publications on MT detection and tracking include $[6,7]$. In [8], MTs are searched in a constrained space for tracking in subsequent frames. In [7], MTs tips are detected by a Gaussian filter and tracked by establishing correspondences in subsequent frames. The proposed approach is sensitive to tips that move out of focus between frames. The authors address this issue by using a graph matching algorithm in [6]. In both [6] and [7], to trace the MT bodies, the authors use active contours that are initialized between the plus end and an estimated point on the MT, found by the Fast Marching (FM) algorithm [10]. The proposed use of FM is sensitive to MT intersections and overlaps as it favors higher intensity levels (caused by intersecting or overlapping MTs) in computing the geodesic distance. In this paper, we provide an algorithm directly addressing this issue.

\section{TRACING MT BODY}

In live cell MT images, intersecting MT regions appear brighter than individual MTs due to additive fluorescence, Fig.2(a). Furthermore, MT segmentation is difficult; typical estimates show gaps on MT bodies, Fig.2(b).

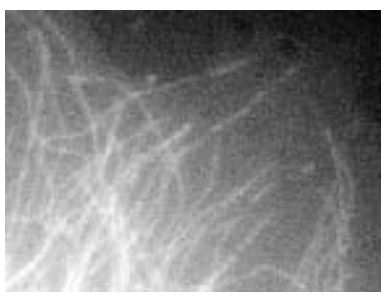

(a)

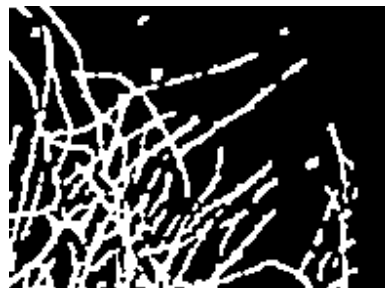

(b)
Fig. 2. (a) Overlapping MTs appear brighter than individual MTs. (b) Binarization of (a) shows gaps on MT bodies.

Consequently, calculating the geodesic with FM on the original or on any grayscale filter response favors overlaps over individual MT bodies in tracing, Fig.3. With high overlaps and intersections among MTs, tracking methods are limited to the single MT segments that appear in isolation. Furthermore, interaction between MTs in subsequent frames may cause the MT body estimation to fail, in which case the primary dynamic parameters are computed erroneously, Fig.4.

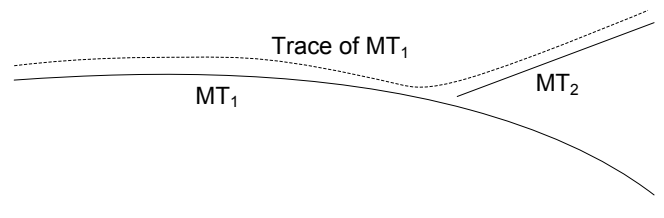

Fig. 3. Diagram of overlapping MTs stealing MT trace.

The problem is the use of FM as a geodesic distance transform, as opposed to determining the shortest path between two known points. We propose to use the geodesic distance between known points, while weighting the paths with a quantity that is (conditionally) independent of the intensity levels along the MT body. Note that performing FM on a binary mask in order to rigidly constrain the geodesic on the MT body would fail whenever there are gaps in the mask along the MT body. However, a binary mask can be used as conditionally independent weights. Thus, if $I(\cdot)$ is the intensity along a MT body, and $B(\cdot)$ denotes the binary transform, we obtain weights $W(\cdot)$ such that the trace extracted by $T($.$) as$ a function of $W$ satisfies $T(W \mid B)=T(W \mid B, I)$.

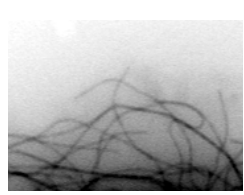

(a)

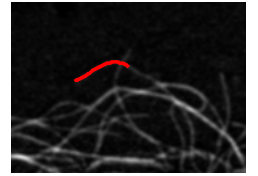

(b)

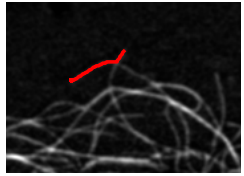

(c)
Fig. 4. Original (a) and consecutive frames with overlaid geodesic MT bodies (b-c), resulting in error in growth. 
Given a MT frame $F(x, y)$, prior to tracing of MT bodies, we first reveal the curvilinear structures by filtering $F(x, y)$ with a second order derivative of Gaussian filter $G_{\sigma, \theta}^{\prime \prime}(x, y)$ with scale $\sigma$ and different orientations $\theta$. Here, note that scale is selected as $\sigma=2$ considering the approximate width of a MT body. Let filter output is represented with $F_{e}(x, y)$.

$$
F_{e}(x, y)=\max _{\theta}\left(F(x, y) * G_{\sigma, \theta}^{\prime \prime}(x, y)\right)
$$

Thinned binary mask, $B(x, y)$, is calculated based on $F_{e}$ which consists of one pixel wide paths representing the coarse estimate of the MT polymers. Line ends on $B(x, y)$ are then marked as tip positions, tip $_{n}=\left(x_{\text {tip }}^{n}, y_{\text {tip }}^{n}\right)$, where $n \in\{1 . . N\}$ and $N$ denotes the number of tip positions.

For each MT tip position, tip $_{n}$, we obtain a $B^{\prime}(x, y)$ by removing $t i p_{n}$ from the $B(x, y)$. Next, we extract all geodesic paths $g p_{n k}$ from the $t i p_{n}$ to the set of all candidate end points $e n d_{k}=\left(x_{e n d}^{k}, y_{\text {end }}^{k}\right), k \in\{1 . . K\}$ such that $\left\{\left(x_{\text {end }}^{k}, y_{\text {end }}^{k}\right): B^{\prime}\left(x_{\text {end }}^{k}, y_{\text {end }}^{k}\right) \neq 0\right\}$.

By this procedure, we formulate the MT tracing as finding the best $g p_{n k}$ at a predefined length. To find the best path, $g p_{n k^{*}}$, we compute the support $s_{n k}$ for $g p_{n k}$ as

$$
s_{n k}=\sum_{(x, y) \in g p_{n k}} B^{\prime}(x, y) .
$$

We declare the path $g p_{n k}$ with maximum support as the initial trace at the predefined length.

$$
g p_{n k^{*}}=\underset{g p_{n k}}{\operatorname{argmax}} s_{n k}
$$

Finally, we use an active contour as described in [6], to adjust the initial trace $g p_{n k^{*}}$ with the MT body. Note that the best path, $g p_{n k^{*}}$, may include sections where it may corresponds to more than one MT in the image. Resolution of this issue is ultimately important in tracking multiple MTs and should be separately addressed in a postprocessing step.

\section{DATASETS}

In this study, we used images from [4] and [5]. In [4], the authors investigate the hypothesis that resistance to taxol may involve altered expression patterns among the different tubulin isotypes. Chinese hamster ovary $(\mathrm{CHO})$ cells were microinjected with rhodamine-labeled tubulin. A total of 111 image sequences were acquired using fluorescence microscopy with 100x objective lens (1000x magnification). 3146 frames were captured at 4 second intervals, from five different conditions. 287 MTs were manually tracked from 65 sequences, with an average of 4.41 MTs per image sequence.

In [5], the authors examine the abilities of different isoforms of the MT-associated protein tau to regulate MT dynamics. The MCF7 human breast cancer cell line was transfected with an EGFP-tubulin plasmid and subsequently microinjected with tau. Images were captured with an inverted fluorescence microscope with a 100x objective lens (1000x magnification). 31 frames were acquired at 4 second intervals from 6 different conditions. A total of 122 image sequences were recorded. 213 MTs were manually tracked from 63 sequences, with an average of 3.38 MTs per image sequence. Further details can be found in respective studies.

For the purposes of evaluating the MT tracing algorithm, we visually selected 8 best sequences from both studies. 32 MTs were manually traced in randomly selected frames. These datasets, as well as the manually extracted traces are available through [11].

\section{RESULTS}

The selection of an appropriate path length is application dependent. For very short lengths, the proposed algorithm would be equivalent to FM. In MT images, very long lengths are not suitable since the minus ends of MTs reach quickly into extreme clutter.

For the tracing accuracy of the suggested method, we evaluated the proposed algorithm against manually traced MTs. The average trace length was obtained from the manual traces. We provide sample results of our algorithm in the typical (Fig.5 - 8) and an ambiguous case (Fig.9) out of the 32 MTs described in the previous section.

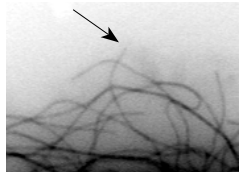

(a)

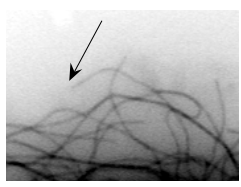

(d)

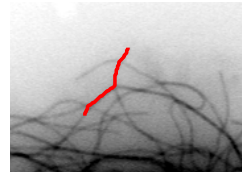

(b)

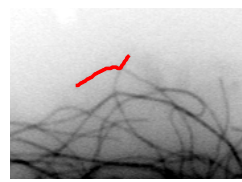

(e)

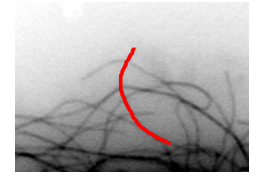

(c)

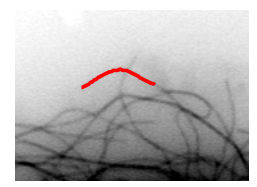

(f)
Fig. 5. Original MTs (a) and (d), results of geodesic paths (b) and (e), and corresponding results of the proposed algorithm (c) and (f).

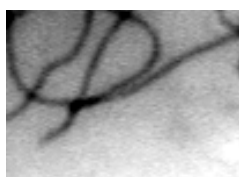

(a)

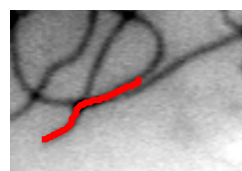

(b)

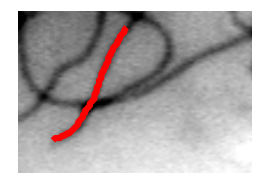

(c)
Fig. 6. Original MTs (a), results of geodesic paths (b), and corresponding results of the proposed algorithm (c).

In Fig.9, while the best path, $g p_{n k^{*}}$, may not correspond to the targeted MT body, the first two $g p_{n k}$ clearly capture 


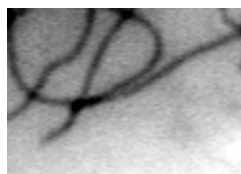

(a)

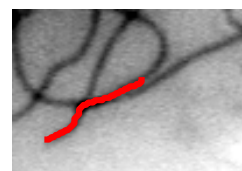

(b)

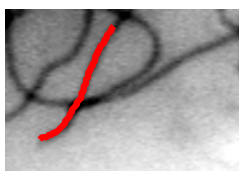

(c)
Fig. 7. Original MTs (a), results of geodesic paths (b), and corresponding results of the proposed algorithm (c).

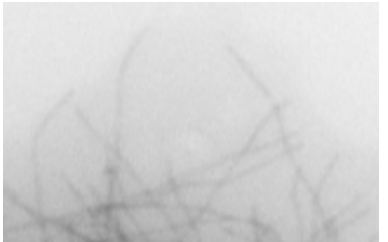

(a)

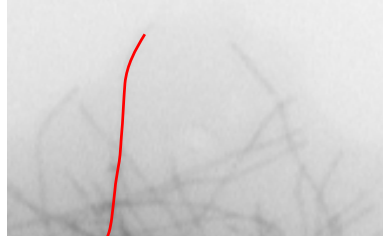

(b)
Fig. 8. Original MTs (a), resulting trace from the proposed algorithm (b).

the trace of the actual MT. In such instances, additional information or use of heuristics may be beneficial to guide the algorithm.

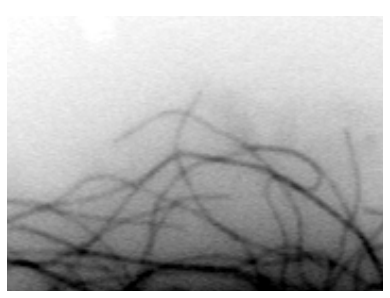

(a)

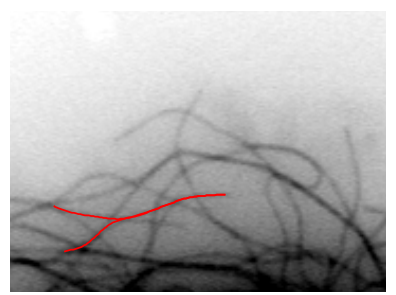

(b)
Fig. 9. Original MT (a) and top two $g p_{n k}$ overlaid (b).

\section{CONCLUSIONS}

The primary indicators of MT dynamics are the growth and the shortening amounts. By tracking only the MT tips, these values are computed as approximations from the change in tip positions, which is inaccurate as compared with the lengths computed along the MT body. Furthermore, a MT body can float and change shape in the cytosol from frame to frame, inflating the approximation error.

With the proposed algorithm, we provide more accurate traces of MT bodies in each frame for tracking the entire MT body. Thus, MT body deformations and displacements can now be quantified over time. Curvature, which is impractical to calculate manually yet biologically significant, can be estimated along the body without applying prior constraints during tracing the MT body. For measuring curvature, correct tracing of the MT is essential.

The proposed algorithm can be applied to other types of data, such as neurons for neurite outgrowth studies or blood vessels, as well as non-biological images for segmentation of thin curvilinear structures. In order to trace multiple MTs simultaneously, overlapping paths may be identified by using the algorithm in a global optimization procedure. MT tracking methods should directly benefit from accurate traces of MT bodies, specifically when temporal information is used to determine overall path consistencies.

\section{REFERENCES}

[1] B. Alberts, A. Johnson, J. Lewis, M. Raff, K. Roberts, and P. Walter, Molecular Biology of the Cell, Garland Scientific, 4th edition edition, 2002.

[2] S. C. Feinstein and L. Wilson, "Inability of tau to properly regulate neuronal microtubule dynamics: a loss-offunction mechanism by which tau might mediate neuronal cell death," Biochimica et Biophysica Acta - Molecular Basis of Disease, vol. 1739, no. 2-3, pp. 268-279, January 2005.

[3] L.D. Cohen and R. Kimmel, "Global minimum for active contour models: A minimal path approach," Int. Jour. of Comp. Vision, vol. 24, no. 1, pp. 57 - 78, 1997.

[4] K. Kamath, L. Wilson, F. Cabral, and M. A. Jordan, " $\beta$ iii-tubulin induces paclitaxel resistance in association with reduced effects on microtubule dynamic instability," The Journal of Biological Chemistry, vol. 280, no. 13, pp. 12902-12907, April 2005.

[5] J. M. Bunker, L. Wilson, M. A. Jordan, and S. C. Feinstein, "Modulation of microtubule dynamics by tau in living cells: Implications for development and neurodegeneration," Molecular Biology of the Cell, vol. 15, no. 6, pp. 2720-2728, June 2004.

[6] A. Altinok et al., "Activity Analysis in Microtubule Videos by Mixture of Hidden Markov Models," CVPR, vol. 2, pp. 1662-1669, 2006.

[7] M. El-Saban et al., "Automated tracking and modeling of microtubule dynamics," ISBI, pp. 1032-1035, 2006.

[8] S. Hadjidemetriou, J. S. Duncan, D. Toomre, and D. Tuck, "Automatic quantification of microtubule dynamics," in Proc. of Int. Symp. on Biomedical Imaging: From Nano to Macro, 2004.

[9] G. Danuser, P.T. Tran, and E.D Salmon, "Tracking differential interference contrast diffraction line images with nanometre sensitivity," Journal of Microscopy, 198, pp. 34-53.

[10] J. Sethian, Level Set Methods and Fast Marching Methods, Cambridge Univ. Press, 1999.

[11] "http://www.bioimage.ucsb.edu," . 